\title{
NOTE ON COMPOSITION OF ENTIRE FUNCTIONS AND BOUNDED $L$-INDEX IN DIRECTION
}

A. I. Bandura, T. M. Salo, O. B. Skaskiv. Note on composition of entire functions and bounded L-index in direction, Mat. Stud. 55 (2021), 51-56.

We study the following question: "Let $f: \mathbb{C} \rightarrow \mathbb{C}$ be an entire function of bounded $l$-index, $\Phi: \mathbb{C}^{n} \rightarrow \mathbb{C}$ an be entire function, $n \geq 2, l: \mathbb{C} \rightarrow \mathbb{R}_{+}$be a continuous function. What is a positive continuous function $L: \mathbb{C}^{n} \rightarrow \mathbb{R}_{+}$and a direction $\mathbf{b} \in \mathbb{C}^{n} \backslash\{\mathbf{0}\}$ such that the composite function $f(\Phi(z))$ has bounded $L$-index in the direction $\mathbf{b}$ ?" In the present paper, early known result on boundedness of $L$-index in direction for the composition of entire functions $f(\Phi(z))$ is modified. We remove a condition that a directional derivative of the inner function $\Phi$ in a direction $\mathbf{b}$ does not equal zero. We relax also the condition $\left|\partial_{\mathbf{b}}^{k} \Phi(z)\right| \leq K\left|\partial_{\mathbf{b}} \Phi(z)\right|^{k}$ for all $z \in \mathbb{C}^{n}$, where $K \geq 1$ is a constant and

$$
\partial_{\mathbf{b}} F(z):=\sum_{j=1}^{n} \frac{\partial F(z)}{\partial z_{j}} b_{j}, \partial_{\mathbf{b}}^{k} F(z):=\partial_{\mathbf{b}}\left(\partial_{\mathbf{b}}^{k-1} F(z)\right)
$$

It is replaced by the condition $\left|\partial_{\mathbf{b}}^{k} \Phi(z)\right| \leq K(l(\Phi(z)))^{1 /(N(f, l)+1)}\left|\partial_{\mathbf{b}} \Phi(z)\right|^{k}$, where $N(f, l)$ is the $l$-index of the function $f$. Under these conditions, the entire function $f(\Phi(z))$ has bounded $L$-index in the direction $\mathbf{b}$ with the function $L(z)=\max \left\{1,\left|\partial_{\mathbf{b}} \Phi(z)\right|\right\} l(\Phi(z))$ satisfying a additional condition. The described result is an improvement of the previous one.

1. Introduction. The present paper is devoted to compositions of entire functions and theory of entire functions of bounded $L$-index in direction. We need some notations and definitions. Let $L: \mathbb{C}^{n} \rightarrow \mathbb{R}_{+}$be any fixed continuous function. An entire function $F(z), z \in$ $\mathbb{C}^{n}$, is called $[1,7,8]$ a function of bounded $L$-index in a direction $\mathbf{b}=\left(b_{1}, \ldots, b_{n}\right) \in \mathbb{C}^{n} \backslash\{\mathbf{0}\}$, if there exists $m_{0} \in \mathbb{Z}_{+}$such that for every $m \in \mathbb{Z}_{+}$and every $z \in \mathbb{C}^{n}$

$$
\frac{\left|\partial_{\mathbf{b}}^{m} F(z)\right|}{m ! L^{m}(z)} \leq \max \left\{\frac{\left|\partial_{\mathbf{b}}^{k} F(z)\right|}{k ! L^{k}(z)}: 0 \leq k \leq m_{0}\right\}
$$

where

$$
\partial_{\mathbf{b}}^{0} F(z):=F(z), \partial_{\mathbf{b}} F(z):=\sum_{j=1}^{n} \frac{\partial F(z)}{\partial z_{j}} b_{j}=\langle\operatorname{grad} F, \overline{\mathbf{b}}\rangle, \partial_{\mathbf{b}}^{k} F(z):=\partial_{\mathbf{b}}\left(\partial_{\mathbf{b}}^{k-1} F(z)\right), k \geq 2 .
$$

The least such integer $m_{0}$ is called the $L$-index in the direction $\mathbf{b}$ and is denoted by $N_{\mathbf{b}}(F, L)$. In the case $n=1$ and $\mathbf{b}=1$ we obtain the definition of an entire function of one variable of

2010 Mathematics Subject Classification: 30D20, 32A15, 32A17.

Keywords: entire function; bounded $L$-index in direction; composite function; bounded $l$-index.

doi:10.30970/ms.55.1.51-56

(c) A. I. Bandura, T. M. Salo, O. B. Skaskiv, 2021 
bounded $l$-index (see $[11,14]$ ) and $N(F, L):=N_{1}(F, L)$; in the case $n=1, \mathbf{b}=1$ and $L(z) \equiv 1$ it is reduced to the definition of a function of bounded index, supposed by B. Lepson [12].

A detailed review of papers on compositions of functions and boundedness of index is presented in [2]. There is considered the following question: Let $f: \mathbb{C} \rightarrow \mathbb{C}$ be an entire function of bounded l-index, $\Phi: \mathbb{C}^{n} \rightarrow \mathbb{C}$ be an entire function, $l: \mathbb{C} \rightarrow \mathbb{R}_{+}$be a continuous function. What are a positive continuous function $L$ and a direction $\mathbf{b} \in \mathbb{C}^{n}$ such that the composite function $f(\Phi(z))$ has bounded L-index in the direction $\mathbf{b}$ ? There is answer to the question as Theorem 1 from [2]. The similar questions are considered for analytic functions in the unit ball [4], for entire functions of bounded L-index in joint variables [3], for entire and analytic functions of bounded $l$ - $M$-index [5], for analytic functions in $\mathbb{C} \times \mathbb{D}[6]$.

Note that the positivity and continuity of the function $L$ are weak restrictions. Therefore, we impose additional conditions by the function $L$.

For $\eta>0, z \in \mathbb{C}^{n}, \mathbf{b}=\left(b_{1}, \ldots, b_{n}\right) \in \mathbb{C}^{n} \backslash\{\mathbf{0}\}$ and a positive continuous function $L: \mathbb{C}^{n} \rightarrow \mathbb{R}_{+}$we define

$$
\lambda(\eta)=\sup _{z \in \mathbb{C}^{n}} \sup _{t}\left\{\frac{L(z+t \mathbf{b})}{L(z)}:|t| \leq \frac{\eta}{L(z)}\right\} .
$$

By $Q_{\mathrm{b}}^{n}$ we denote the class of functions $L$ such that $\lambda(\eta)$ is finite for any $\eta>0$. We also use notation $Q=Q_{1}^{1}$ for the class of positive continuous function $l(z)$, when $z \in \mathbb{C}, \mathbf{b}=1$, $n=1, L \equiv l$.

To prove the main theorem we need auxiliary proposition.

Lemma 1 ([1,7]). Let $\mathbf{b} \in \mathbb{C}^{n} \backslash\{\mathbf{0}\}$ and $L \in Q_{\mathbf{b}}^{n}$. An entire function $F(z)$ has bounded $L$-index in the direction $\mathbf{b}$ if and only if there exist numbers $p \in \mathbb{Z}_{+}, R>0$ and $C>0$ such that for each $z \in \mathbb{C}^{n},|z| \geq R$,

$$
\frac{\left|\partial_{\mathbf{b}}^{p+1} F(z)\right|}{L^{p+1}(z)} \leq C \max \left\{\frac{\left|\partial_{\mathbf{b}}^{k} F(z)\right|}{L^{k}(z)}: 0 \leq k \leq p\right\} .
$$

There was obtained the following result

Proposition $1([2])$. Let $\mathbf{b} \in \mathbb{C}^{n} \backslash\{\mathbf{0}\}, f$ be an entire function in $\mathbb{C}, \Phi$ be an entire function in $\mathbb{C}^{n}$ such that $\partial_{\mathbf{b}} \Phi(z) \neq 0$ and

$$
\left|\partial_{\mathbf{b}}^{j} \Phi(z)\right| \leq K\left|\partial_{\mathbf{b}} \Phi(z)\right|^{j}, \quad K \equiv \text { const }>0,
$$

for all $z \in \mathbb{C}^{n}$ and every $j \leq p$, where $p$ is defined in (2).

Let $l \in Q, l(w) \geq 1, w \in \mathbb{C}$ and $L \in Q_{\mathbf{b}}^{n}$, where $L(z)=\left|\partial_{\mathbf{b}} \Phi(z)\right| l(\Phi(z))$. The entire function $f$ has bounded l-index if and only if $F(z)=f(\Phi(z))$ has bounded L-index in the direction $\mathbf{b}$.

Note that the conditions for every $j \in\{1, \ldots, p\} \quad \partial_{\mathbf{b}} \Phi(z) \neq 0$ and $\left|\partial_{\mathbf{b}}^{j} \Phi(z)\right| \leq K\left|\partial_{\mathbf{b}} \Phi(z)\right|^{j}$, in Proposition 1 are generated by the method of proof. In fact, we can remove it and prove more general proposition with some greater function $L$.

Our main result is following.

Theorem 1. Let $l \in Q, f: \mathbb{C} \rightarrow \mathbb{C}$ be an entire function of bounded l-index, $\Phi: \mathbb{C}^{n} \rightarrow \mathbb{C}$ be an entire function, $\mathbf{b} \in \mathbb{C}^{n} \backslash\{\mathbf{0}\}$. Suppose that $l(w) \geq 1, w \in \mathbb{C}$, and $L \in Q_{\mathbf{b}}^{n}$ with

$$
L(z)=\max \left\{1,\left|\partial_{\mathbf{b}} \Phi(z)\right|\right\} l(\Phi(z))
$$


and for all $z \in \mathbb{C}^{n}$ and $k \in\{1,2, \ldots, N(f, l)+1\}$ one has

$$
\left|\partial_{\mathbf{b}}^{k} \Phi(z)\right| \leq K(l(\Phi(z)))^{1 /(N(f, l)+1)}\left|\partial_{\mathbf{b}} \Phi(z)\right|^{k}
$$

where $K \geq 1$ is a constant. Then the entire function $F(z)=f(\Phi(z))$ has bounded $L$-index in the direction $\mathbf{b}$.

Theorem 1 is new even in one-dimensional case, i.e. for entire functions of one variable having bounded $l$-index.

2. Proof of main Theorem. For $n=1$ Lemma 1 is Sheremeta's result [13]. W. K. Hayman [10] proved Lemma 1 for entire functions of bounded index. Analogs of the Hayman Theorem are also known for other classes of holomorphic functions of bounded index $[9,13,14]$.

Proof of Theorem 1. In the proof of Proposition 1 in [2] there was established the following formula by the method of mathematical induction

$$
\partial_{\mathbf{b}}^{k} F(z)=f^{(k)}(\Phi(z))\left(\partial_{\mathbf{b}} \Phi(z)\right)^{k}+\sum_{j=1}^{k-1} f^{(j)}(\Phi(z)) Q_{j, k}(z)
$$

where

$$
Q_{j, k}(z)=\sum_{\substack{n_{1}+2 n_{2}+\ldots+k n_{k}=k \\ 0 \leq n_{1} \leq j-1}} c_{j, k, n_{1}, \ldots, n_{k}}\left(\partial_{\mathbf{b}} \Phi(z)\right)^{n_{1}}\left(\partial_{\mathbf{b}}^{2} \Phi(z)\right)^{n_{2}} \ldots\left(\partial_{\mathbf{b}}^{k} \Phi(z)\right)^{n_{k}},
$$

and $c_{j, k, n_{1}, \ldots, n_{k}}$ are some non-negative integer coefficients. There was also deduced that

$$
f^{(k)}(\Phi(z))=\frac{\partial_{\mathbf{b}}^{k} F(z)}{\left(\partial_{\mathbf{b}} \Phi(z)\right)^{k}}+\frac{1}{\left(\partial_{\mathbf{b}} \Phi(z)\right)^{2 k}} \sum_{j=1}^{k-1} \partial_{\mathbf{b}}^{j} \Phi(z)\left(\partial_{\mathbf{b}} \Phi(z)\right)^{j} Q_{j, k}^{*}(z),
$$

where

$$
Q_{j, k}^{*}(z)=\sum_{m_{1}+2 m_{2}+\ldots+k m_{k}=2(k-j)} b_{j, k, m_{1}, \ldots, m_{k}}\left(\partial_{\mathbf{b}} \Phi(z)\right)^{m_{1}}\left(\partial_{\mathbf{b}}^{2} \Phi(z)\right)^{m_{2}} \ldots\left(\partial_{\mathbf{b}}^{k} \Phi(z)\right)^{m_{k}},
$$

and $b_{j, k, m_{1}, \ldots, m_{k}}$ are some integer coefficients.

Let $f$ be an entire function of bounded $l$-index. Denote $L_{0}(z)=l(\Phi(z))\left|\partial_{\mathbf{b}} \Phi(z)\right|$. Taking into account (6) and (4), for $k=p+1$ we have

$$
\begin{aligned}
& \frac{\left|\partial_{\mathbf{b}}^{p+1} F(z)\right|}{L_{0}^{p+1}(z)} \leq \frac{\left|f^{(p+1)}(\Phi(z))\right|}{L_{0}^{p+1}(z)}\left|\partial_{\mathbf{b}} \Phi(z)\right|^{p+1}+\sum_{j=1}^{p} \frac{\left|f^{(j)}(\Phi(z))\right|\left|Q_{j, p+1}(z)\right|}{L_{0}^{p+1}(z)} \leq \\
& \leq \frac{\left|f^{(p+1)}(\Phi(z))\right|\left|\partial_{\mathbf{b}} \Phi(z)\right|^{p+1}}{l^{p+1}(\Phi(z))\left|\partial_{\mathbf{b}} \Phi(z)\right|^{p+1}}+\sum_{j=1}^{p} \frac{\left|f^{(j)}(\Phi(z))\right|}{l^{j}(\Phi(z))} \cdot \frac{\left|Q_{j, p+1}(z)\right| l^{j}(\Phi(z))}{\left|\partial_{\mathbf{b}} \Phi(z)\right|^{p+1} l^{p+1}(\Phi(z))} .
\end{aligned}
$$

By Lemma 1 inequality (2) is valid for $n=1, F=f, L=l, \mathbf{b}=1$ and $p=N(f, l)$.

$$
(\forall \tau \in \mathbb{C}): \quad \frac{\left|f^{(p+1)}(\tau)\right|}{l^{p+1}(\tau)} \leq C \max \left\{\frac{\left|f^{(k)}(\tau)\right|}{l^{k}(\tau)}: 0 \leq k \leq p\right\} .
$$


Applying to (8) this inequalities with $\tau=\Phi(z)$, we obtain

$$
\begin{aligned}
& \frac{\left|\partial_{\mathbf{b}}^{p+1} F(z)\right|}{L_{0}^{p+1}(z)} \leq \max \left\{\frac{\left|f^{(k)}(\Phi(z))\right|}{l^{k}(\Phi(z))}: 0 \leq k \leq p\right\}\left(C+\sum_{j=1}^{p} \frac{\left|Q_{j, p+1}(z)\right| l^{j-p-1}(\Phi(z))}{\left|\partial_{\mathbf{b}} \Phi(z)\right|^{p+1}}\right) \leq \\
& \leq \max \left\{\frac{\left|f^{(k)}(\Phi(z))\right|}{l^{k}(\Phi(z))}: 0 \leq k \leq p\right\}(C+ \\
& \left.+\sum_{j=1}^{p} \sum_{\substack{n_{1}+2 n_{2}+\ldots+(p+1) n_{p+1}=p+1 \\
0 \leq n_{1} \leq j-1}} c_{j, p+1, n_{1}, \ldots, n_{p+1}} \frac{\left|\left(\partial_{\mathbf{b}} \Phi(z)\right)^{n_{1}}\left(\partial_{\mathbf{b}}^{2} \Phi(z)\right)^{n_{2}} \ldots\left(\partial_{\mathbf{b}}^{p+1} \Phi(z)\right)^{n_{p+1}}\right|}{l^{p+1-j}(\Phi(z))\left|\partial_{\mathbf{b}} \Phi(z)\right|^{p+1}}\right) .
\end{aligned}
$$

In view of condition (5) inequality (9) yields

$$
\begin{aligned}
& \frac{\left|\partial_{\mathbf{b}}^{p+1} F(z)\right|}{L_{0}^{p+1}(z)} \leq \max \left\{\frac{\left|f^{(k)}(\Phi(z))\right|}{l^{k}(\Phi(z))}: 0 \leq k \leq p\right\} \times \\
& \times\left(C+\sum_{j=1}^{p} \sum_{\substack{n_{1}+2 n_{2}+\ldots+(p+1) n_{p+1}=p+1 \\
0 \leq n_{1} \leq j-1}} \frac{c_{j, p+1, n_{1}, \ldots, n_{p+1}} K^{p+1} l(\Phi(z))\left|\partial_{\mathbf{b}} \Phi(z)\right|^{p+1}}{l^{p+1-j}(\Phi(z))\left|\partial_{\mathbf{b}} \Phi(z)\right|^{p+1}}\right) \leq \\
& \leq \max \left\{\frac{\left|f^{(k)}(\Phi(z))\right|}{l^{k}(\Phi(z))}: 0 \leq k \leq p\right\}\left(C+\sum_{j=1_{n_{1}+2 n_{2}+\ldots+(p+1) n_{p+1}=p+1}}^{p} \sum_{\substack{0 \leq n_{1} \leq j-1}} \frac{c_{j, p+1, n_{1}, \ldots, n_{p+1}} K^{p+1}}{l^{p-j}(\Phi(z))}\right) .
\end{aligned}
$$

We will use that $l(\Phi(z)) \geq 1$. Then from (10) it follows that

$$
\frac{\left|\partial_{\mathbf{b}}^{p+1} F(z)\right|}{L_{0}^{p+1}(z)} \leq C_{1} \max \left\{\frac{\left|f^{(k)}(\Phi(z))\right|}{l^{k}(\Phi(z))}: 0 \leq k \leq p\right\},
$$

where

$$
C_{1}=C+K^{p+1} \sum_{j=1}^{p} \sum_{\substack{n_{1}+2 n_{2}+\ldots+(p+1) n_{p+1}=p+1 \\ 0 \leq n_{1} \leq j-1}} c_{j, p+1, n_{1}, \ldots, n_{p+1}} .
$$

Applying equality (7), we can estimate the fraction $\frac{\left|f^{(k)}(\Phi(z))\right|}{l^{k}(\Phi(z))}$

$$
\begin{aligned}
& \frac{\left|f^{(k)}(\Phi(z))\right|}{l^{k}(\Phi(z))} \leq \frac{\left|\partial_{\mathbf{b}}^{k} F(z)\right|}{l^{k}(\Phi(z))\left|\partial_{\mathbf{b}} \Phi(z)\right|^{k}}+\sum_{j=1}^{k-1} \frac{\left|\partial_{\mathbf{b}}^{j} F(z)\right|\left|Q_{j, k}^{*}(z)\right|}{l^{k}(\Phi(z))\left|\partial_{\mathbf{b}} \Phi(z)\right|^{2 k-j}} \leq \\
& \leq \max \left\{\frac{\left|\partial_{\mathbf{b}}^{j} \Phi(z)\right|}{l^{j}(\Phi(z))\left|\partial_{\mathbf{b}} \Phi(z)\right|^{j}}: 1 \leq j \leq k\right\}\left(1+\sum_{j=1}^{k-1} \frac{\left|Q_{j, k}^{*}(z)\right|}{l^{k-j}(\Phi(z))\left|\partial_{\mathbf{b}} \Phi(z)\right|^{2(k-j)}}\right) \leq \\
& \leq \max \left\{\frac{\left|\partial_{\mathbf{b}}^{j} \Phi(z)\right|}{l^{j}(\Phi(z))\left|\partial_{\mathbf{b}} \Phi(z)\right|^{j}}: 1 \leq j \leq k\right\}(1+ \\
& \left.+\sum_{j=1}^{k-1} \sum_{m_{1}+2 m_{2}+\ldots+k m_{k}=2(k-j)}\left|b_{j, k, m_{1}, \ldots, m_{k}}\right| \frac{\left|\left(\partial_{\mathbf{b}} \Phi(z)\right)^{m_{1}}\left(\partial_{\mathbf{b}}^{2} \Phi(z)\right)^{m_{2}} \ldots\left(\partial_{\mathbf{b}}^{k} \Phi(z)\right)^{m_{k}}\right|}{l^{k-j}(\Phi(z))\left|\partial_{\mathbf{b}} \Phi(z)\right|^{2(k-j)}}\right) .
\end{aligned}
$$

From inequalities (5) and $l(w) \geq 1$ it follows that $\left|\partial_{\mathbf{b}}^{s} \Phi(z)\right| \leq K l^{s / 2}(\Phi(z))\left|\partial_{\mathbf{b}} \Phi(z)\right|^{s}$, because $s / 2 \geq 1 /(N(f, l)+1)$ for $s \in\{1,2, \ldots, N(f, l)+1\}$. Applying this inequality to (12), we 
deduce

$$
\begin{gathered}
\frac{\left|f^{(k)}(\Phi(z))\right|}{l^{k}(\Phi(z))} \leq \max \left\{\frac{\left|\partial_{\mathbf{b}}^{j} F(z)\right|}{l^{j}(\Phi(z))\left|\partial_{\mathbf{b}} \Phi(z)\right|^{j}}: 1 \leq j \leq k\right\}\left(1+\sum_{j=1}^{k-1} \sum_{m_{1}+2 m_{2}+\ldots+k m_{k}=2(k-j)} \times\right. \\
\left.\times\left|b_{j, k, m_{1}, \ldots, m_{k}}\right| K^{m_{1}+m_{2}+\ldots+m_{k}} \frac{(l(\Phi(z)))^{\left(m_{1}+2 m_{2}+\ldots+k m_{k}\right) / 2}\left|\partial_{\mathbf{b}} \Phi(z)\right|^{m_{1}+2 m_{2}+\ldots+k m_{k}}}{l^{k-j}(\Phi(z))\left|\partial_{\mathbf{b}} \Phi(z)\right|^{2(k-j)}}\right) \leq \\
\leq C_{2} \max \left\{\frac{\left|\partial_{\mathbf{b}}^{j} \Phi(z)\right|}{l^{j}(\Phi(z))\left|\partial_{\mathbf{b}} \Phi(z)\right|^{j}}: 1 \leq j \leq k\right\},
\end{gathered}
$$

where

$$
C=1+\sum_{j=1}^{k-1} \sum_{m_{1}+2 m_{2}+\ldots+k m_{k}=2(k-j)}\left|b_{j, k, m_{1}, \ldots, m_{k}}\right| K^{m_{1}+m_{2}+\ldots+m_{k}} .
$$

Then from inequality (11) we get

$$
\frac{\left|\partial_{\mathbf{b}}^{p+1} F(z)\right|}{L_{0}^{p+1}(z)} \leq C_{1} \max \left\{\frac{\left|f^{(k)}(\Phi(z))\right|}{l^{k}(\Phi(z))}: 0 \leq k \leq p\right\} \leq C_{1} C_{2} \max \left\{\frac{\left|\partial_{\mathbf{b}}^{j} F(z)\right|}{L_{0}^{j}(z)}: 0 \leq j \leq p\right\},
$$

$p=N(f, l)$. The last inequality is proved for all $z$ such that $\partial_{\mathbf{b}} \Phi(z) \neq 0$.

Remind that $L(z)=l(\Phi(z)) \max \left\{1,\left|\partial_{\mathbf{b}} \Phi(z)\right|\right\}$. Rewrite inequality (13) in the following form:

$$
\frac{\left|\partial_{\mathbf{b}}^{p+1} F(z)\right|}{L^{p+1}(z)} \cdot \frac{L^{p+1}(z)}{L_{0}^{p+1}(z)} \leq C_{1} C_{2} \max \left\{\frac{\left|\partial_{\mathbf{b}}^{k} F(z)\right|}{L^{k}(z)} \frac{L^{k}(z)}{L_{0}^{k}(z)}: 0 \leq k \leq p\right\} .
$$

Then

$$
\begin{gathered}
\frac{\left|\partial_{\mathbf{b}}^{p+1} F(z)\right|}{L^{p+1}(z)} \leq C_{1} C_{2} \frac{L_{0}^{p+1}(z)}{L^{p+1}(z)} \max \left\{\frac{\left|\partial_{\mathbf{b}}^{k} F(z)\right|}{L^{k}(z)} \frac{L^{k}(z)}{L_{0}^{k}(z)}: 0 \leq k \leq p\right\} \leq \\
\leq C_{1} C_{2} \frac{L_{0}^{p+1}(z)}{L^{p+1}(z)} \max \left\{\frac{\left|\partial_{\mathbf{b}}^{k} F(z)\right|}{L^{k}(z)}: 0 \leq k \leq p\right\} \max \left\{\frac{L^{k}(z)}{L_{0}^{k}(z)}: 0 \leq k \leq p\right\}= \\
=C_{1} C_{2} \frac{\left(L_{0}(z) / L(z)\right)^{p+1}}{\min _{0 \leq k \leq p}\left(L_{0}(z) / L(z)\right)^{k}} \max \left\{\frac{\left|\partial_{\mathbf{b}}^{k} F(z)\right|}{L^{k}(z)}: 0 \leq k \leq p\right\} .
\end{gathered}
$$

Let $t_{0}=t(z)=L_{0}(z) / L(z)$ and $k_{0} \leq p\left(k_{0} \in \mathbb{Z}_{+}\right)$be such that $\left(t_{0}\right)^{k_{0}}=\min _{0 \leq k \leq p} t_{0}^{k}$. One should observe that $t_{0} \in(0,1]$ and $p+1-k_{0} \geq 1$. Hence, $\frac{t_{0}^{p+1}}{t_{0}^{k_{0}}}=t_{0}^{p+1-k_{0}} \leq t_{0} \leq 1$. Therefore,

$$
\frac{\left(L_{0}(z) / L(z)\right)^{p+1}}{\min _{0 \leq k \leq p}\left(L_{0}(z) / L(z)\right)^{k}}=t_{0}^{p+1-k_{0}} \leq t_{0} \leq 1
$$

Thus, from inequality (14) we get

$$
\frac{\left|\partial_{\mathbf{b}}^{p+1} F(z)\right|}{L^{p+1}(z)} \leq C_{1} C_{2} \max \left\{\frac{\left|\partial_{\mathbf{b}}^{k} F(z)\right|}{L^{k}(z)}: 0 \leq k \leq p\right\}
$$

for all $z$ such that $\partial_{\mathbf{b}} \Phi(z) \neq 0$. 
If $\partial_{\mathbf{b}} \Phi(z)=0$ then for any $k \in\{1, \ldots, N(f, \ell)+1\}$ inequality (5) implies $\partial_{\mathbf{b}}^{k} \Phi(z)=0$. In view of (6) it means that for each $k \in\{1, \ldots, N(f, l)+1\} \quad \partial_{\mathbf{b}}^{k} F(z)=0$. Thus, for the points $z$ such that $\partial_{\mathbf{b}} \Phi(z)=0$ inequality (15) is also satisfied.

Therefore, by Lemma 1 this inequality means that the function $F$ has bounded $L$-index in the direction $\mathbf{b}$.

Acknowledgments. The research of the first author was funded by the National Research Foundation of Ukraine, 2020.02/0025, 0120U103996.

\section{REFERENCES}

1. A.I. Bandura, O.B. Skaskiv, Entire functions of bounded L-index in direction, Mat. Stud., 27 (2007), №1, 30-52. (in Ukrainian)

2. A. Bandura, Composition of entire functions and bounded L-index in direction, Mat. Stud., 47 (2017), №2, 179-184. doi: 10.15330/ms.47.2.179-184

3. A.I Bandura, O.B. Skaskiv, Boundedness of L-index for the composition of entire functions of several variables, Ukr. Math. J., 70 (2019), №10, 1538-1549. doi: 10.1007/s11253-019-01589-9

4. A.I Bandura, Composition, product and sum of analytic functions of bounded L-index in direction in the unit ball, Mat. Stud., 50 (2018), №2, 115-134. doi: 10.15330/ms.50.2.115-134

5. A.I. Bandura, M.M. Sheremeta, Bounded $l$-index and $l$-M-index and compositions of analytic functions, Mat. Stud., 48 (2017), №2, 180-188. doi: 10.15330/ms.48.2.180-188

6. A.I. Bandura, O.B. Skaskiv, V.L. Tsvigun, The functions of bounded L-index in the collection of variables analytic in $\mathbb{D} \times \mathbb{C}$, J. Math. Sci., 246 (2020), №2, 256-263. doi: 10.1007/s10958-020-04735-y

7. A. Bandura, O. Skaskiv, Entire functions of several variables of bounded index, Lviv: Publisher I. E. Chyzhykov, 2016, 128 p.

8. A. Bandura, O. Skaskiv, P. Filevych, Properties of entire solutions of some linear PDE's, J. Appl. Math. Comput. Mech., 16 (2017), №2, 17-28. doi:10.17512/jamcm.2017.2.02

9. A. Bandura, N. Petrechko, O. Skaskiv, Maximum modulus in a bidisc of analytic functions of bounded L-index and an analogue of Hayman's theorem, Mat. Bohemica, 143 (2018), №4, 339-354. doi: 10.21136/MB.2017.0110-16

10. W.K. Hayman, Differential inequalities and local valency, Pacific J. Math., 44 (1973), №1, 117-137.

11. A.D. Kuzyk, M.N. Sheremeta, Entire functions of bounded l-distribution of values, Mat. Zametki, 39 (1986), №1, 3-13. Engl. transl.: Math. Notes, 39 (1986), №1, 3-8. doi:10.1007/BF01647624

12. B. Lepson, Differential equations of infinite order, hyperdirichlet series and entire functions of bounded index, Proc. Sympos. Pure Math., 11 (1968), 298-307.

13. M.N. Sheremeta, Entire functions and Dirichlet series of bounded l-index, Russian Math. (Iz. VUZ), 36 (1992), №9, 76-82.

14. M. Sheremeta, Analytic functions of bounded index, Lviv: VNTL Publishers, 1999, 141 p.

Ivano-Frankivsk National Technical University of Oil and Gas

Ivano-Frankivsk, Ukraine

andriykopanytsia@gmail.com

Lviv Politechnic National University

Lviv, Ukraine

tetyan.salo@gmail.com

Ivan Franko National University of Lviv

Lviv, Ukraine

olskask@gmail.com 